\title{
Is treatment with ICS and LABA cost-effective for COPD? Multinational economic analysis of the TORCH study
}

\author{
A.H. Briggs*\#, H.A. Glick, G. Lozano-Ortega", M. Spencer ${ }^{+}$, P.M.A. Calverley ${ }^{\S}$, \\ P.W. Jones ${ }^{f}$ and J. Vestbo***\# on behalf of the TOwards a Revolution in COPD \\ Health (TORCH) investigators
}

ABSTRACT: The TOwards a Revolution in COPD Health (TORCH) study was a 3-yr multicentre trial of 6,112 patients randomised to salmeterol (Salm), fluticasone propionate (FP), a Salm/FP combination (SFC) or placebo (P). Here the cost-effectiveness of treatments evaluated in the TORCH study is assessed.

For four regions, 3-yr all-cause hospitalisation, medication and outpatient care costs were calculated. The sample was restricted to the 21 countries $(n=4,237)$ in which European quality of life five-dimension (EQ-5D) data were collected in order to estimate the number of qualityadjusted life years (QALYs). Regression models were fitted to survival, study medication cost, other medication cost and EQ-5D data in order to estimate total cost, number of QALYs and cost per QALY, adjusted for missing data and region.

SFC had a trial-wide estimate of cost per QALY of 43,600 US dollars (USD) compared with P (95\% confidence interval 21,400-123,500 USD). Estimates for Salm versus $P$ (197,000 USD) and FP versus $P$ (78,000 USD) were less favourable. The US estimates were greater than those from other regions; for SFC versus $P$, the cost per QALY was $77,100(46,200-241,700)$ USD compared to $24,200(15,200-56,100)$ USD in Western Europe.

Compared with P, SFC has a lower incremental cost-effectiveness ratio than either FP or Salm used alone, and is, therefore, preferred to these monotherapies on the grounds of costeffectiveness.

KEYWORDS: Chronic obstructive pulmonary disease, cost analysis, cost-effectiveness analysis, quality of life, randomised trials

hronic obstructive pulmonary disease (COPD) is an incurable, debilitating and progressive disease that can be fatal. Patients typically suffer a prolonged respiratory and systemic functional decline punctuated by acute exacerbations often requiring hospital treatment [1]. The incidence of COPD is increasing world wide, making it one of the greatest disease burdens in many countries [2]. Pharmacological treatments that can extend life and improve the quality of life of COPD patients have the potential to offset this burden. Nevertheless, if they are to become routinely available, health systems around the world need evidence of not only the effectiveness but also the cost-effectiveness of such interventions.

Despite the importance of COPD as a major disease burden, there is a paucity of high-quality costeffectiveness data concerning potential treatments.
The TOwards a Revolution in COPD Health (TORCH) study has recently reported its clinical findings [3]. This multinational 3-yr study was the first trial of pharmacotherapy to adopt mortality as a primary end-point in COPD, and included a rigorous tracking of deaths in order to ensure complete follow-up. Furthermore, the TORCH study prospectively collected data on medical resource use and health-related quality of life for patients in the trial. This provides an opportunity to assess the impact of study treatments on health economic outcomes in order to judge whether or not they are worthwhile from the perspective of the healthcare system.

The aim of the present article is to report the analysis of the economic data from the TORCH study in order to inform decision-makers of the potential cost-effectiveness of alternative treatments for COPD. Special consideration is given to
AFFILIATIONS

*University of Glasgow, Glasgow, +Janssen-Cilag, High Wycombe, ${ }^{\S}$ University Hospital Aintree, Liverpool,

${ }^{f}$ St George's Medical School, University of London, London, **Wythenshawe Hospital, Manchester, UK,

- University of Pennsylvania, Philadelphia, PA, USA,

\#Oxford Outcomes (Canada), Vancouver, BC, Canada, and \#\#Hvidovre Hospital, Hvidovre, Denmark.

\section{CORRESPONDENCE}

A. Briggs

Section of Public Health \&

Health Policy

University of Glasgow

1 Lilybank Gardens

Glasgow

G12 8RZ

UK

E-mail: a.briggs@clinmed.gla.ac.uk

Received:

Oct 182008

Accepted after revision:

Aug 192009

First published online:

Aug 282009 
the need to estimate cost-effectiveness results that are relevant to decision-makers in particular jurisdictions covered by this multinational trial.

\section{METHODS \\ The trial}

The TORCH study was a multicentric randomised doubleblind parallel-group placebo (P)-controlled study to investigate the long-term effects of the long-acting $\beta_{2}$-agonist salmeterol (Salm) $50 \mu \mathrm{g}$ and the inhaled corticosteroid fluticasone propionate (FP) $500 \mu \mathrm{g}$, individually and as a fixed-dose combination (SFC), relative to $\mathrm{P}$, on the survival of participants with COPD during 3 yrs of treatment. Recruited patients had a diagnosis of COPD with a forced expiratory volume in $1 \mathrm{~s}$ (FEV1) of $<60 \%$ of the predicted value [4], had a smoking history of $\geqslant 10$ pack-yrs and were aged $40-80$ yrs. The study was conducted in 42 countries in five regions (the USA, Eastern Europe, Western Europe, Asia/Pacific and other).

\section{End-points}

The primary end-point was all-cause mortality. Data were also collected on health status and medical resource use. Health status was measured using the European quality of life fivedimension (EQ-5D) questionnaire [5] at baseline, every 24 weeks during the study and at the final visit after 3 yrs of follow-up. Preference weights (utilities) for EQ-5D health states were derived using the recommended tariffs derived from a UK population survey [6]. EQ-5D data were only collected in the 21 of the 42 participating countries for which validated translations of the instrument were available, so the cost-effectiveness analysis was limited to trial participants in these 21 countries (which enrolled 4,237 of the total 6,112 study participants). Since none of the seven Asian/Pacific countries collected EQ-5D data, this region was excluded from the costeffectiveness analysis.

Recorded medical resource use included: study medication, COPD hospitalisation (days in intensive care units/routine care), non-COPD hospitalisation (number of admissions), physician visits for COPD (including outpatient and general practitioner appointments), telephone contacts for COPD, long-term oxygen therapy and concomitant medications (both COPD and non-COPD-related). Non-COPD-related physician visits and any rehabilitation services used were not recorded. Costs borne by patients or their carers and productivity losses to society were considered outside the scope of the health service/third-party payer perspective of the present analysis.

\section{Price weights}

Price weights (i.e. unit costs) for the three study medications were compiled from the IMS Health (Norwalk, CT, USA) database [7] for all 21 of the countries that were included in the analysis. Price weights for COPD hospitalisations, outpatient care and concomitant medications were obtained for 12 of the 21 countries and weights for non-COPD hospitalisations were obtained for 11 countries. These countries were chosen so as to include representatives from the four main regions that participated in the trial. For the remaining countries, the mean price weights from countries with a similar level of development (e.g. developed or developing) were used. Price weights were translated to a common currency (US dollars (USD)) by use of purchasing power parity statistics reported by the
Organization for Economic Co-operation and Development (OECD) for 2006 [8]. The exceptions were Argentina and South Africa, for which no OECD purchasing power parity was available and for which other sources were used $[9,10]$. Where necessary, costs were inflated to the 2007 base year.

\section{Other explanatory variables}

Other explanatory variables included in the statistical models were age, body mass index, race, sex, count of items in medical history at baseline (as an indicator of high medical resource use), count of pre-randomisation exacerbations requiring hospitalisation, baseline percentage predicted FEV1, UK Medical Research Council dyspnoea score [11], disease-specific health status (St George's Respiratory Questionnaire [12]) and region. As a smoking history of 10 pack-yrs was an inclusion criterion for the trial, all participants were current or previous smokers at study entry, and this baseline smoking status was also included.

\section{Analysis}

Figure 1 provides an overview of the methods used to estimate the components of the cost-effectiveness calculation. In the first stage, multivariate regression models were fitted separately for survival, study medication cost, other medical cost and preference data. In the second stage, estimates of these four components were combined to give total cost, QALY number and cost-per-QALY estimates. This approach is described in more detail below, with particular emphasis on the methods for handling missing data due to attrition, which affected the secondary end-points of medical resource use and health status, and the adjustment of the estimates to the regions of the study.

\section{Missing data}

There were no missing survival data, except for 1 yr of followup in one subject [3]. Withdrawal from the study or missed follow-up visits resulted in $22.6 \%$ of the medical service use data and $26.1 \%$ of the EQ-5D data being missing. This missingness was addressed by use of inverse probability weighting $[13,14]$. Weights were defined as the reciprocal of the estimated probability of being observed (generated from logistic regression with missingness status as the dependent variable) and then utilised in weighted regression models to estimate study medication cost, other medical cost and preference scores adjusted for the missing values. Further details relating to the modelling of each component of the costeffectiveness calculation are given below.

\section{Survival}

Mortality was estimated as a function of explanatory prognostic factors and treatment allocation by use of a parametric Weibull survival model. The number of life years associated with each treatment arm (overall and as a function of other important prognostic factors, including geographical region) were estimated as the area under the Weibull curve.

\section{Cost}

Separate estimates of the cost of study medication and other costs were made, recognising that the treatment cost was part of the trial protocol, whereas other costs related to the health system. Generalised linear models were used to estimate study 
Stage 1: modelling

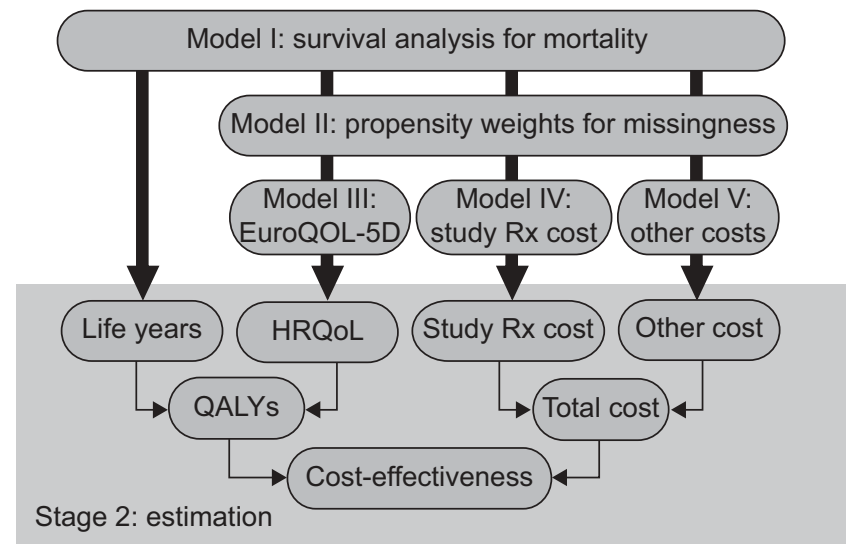

FIGURE 1. Schematic of analyses. In the first stage, multivariate regression models were fitted to the TOwards a Revolution in COPD Health (TORCH) data, with treatment and region as covariates (alongside other prognostic factors). In the first model, a parametric (Weibull) time-to-event model was fitted directly to the mortality data since these data were complete. Owing to attrition, there were missing data in the preference (European quality of life five-dimension (EQ-5D)) and cost data; therefore, the second model estimated a set of weights based on the estimated propensity for observations to be missing via logistic regression. These weights were then used in weighted regression analyses of the preference and cost data in models III, IV and V. In the second stage, the fitted models were used to estimate the components of the cost-effectiveness calculation. The health-related quality of life (HRQoL) preference estimates were combined with the number of life years to estimate the number of quality-adjusted life years (QALYS), and the study medication $(\mathrm{Rx})$ and other medical service use costs were combined to estimate total cost.

medication cost and other costs, utilising separate link functions and families guided by the fit to the data [15].

\section{Preferences}

Ordinary least squares regression was employed to model the EQ-5D scores. The number of QALYs in each treatment arm were calculated by weighting the per-period estimates of preference scores by the probability of survival at the corresponding visit.

\section{Regional estimates}

The TORCH study included participants from five geographic/economic regions of the world: the USA, Western Europe, Eastern Europe, Asia/Pacific and all other countries. Since no validated EQ-5D instrument was available for the languages in the Asian/Pacific region, the economic analysis presented here relates only to the remaining four regions. Regional estimates of costs and number of QALYs for each of the four arms of the TORCH study were generated by including regional main effect terms alongside the treatment indicators and other explanatory variables in each of the four regression models described above. Treatment-by-region interaction terms were evaluated for each regression, but these terms were only included in the final regression model if the joint test of significance gave a p-value of $<0.05$. Treatment, region and treatment-by-region interaction terms were estimated taking into account the baseline characteristics of subjects in that region. It should be noted that the economic results are aggregated and presented at the regional rather than the country level since the number of patients recruited by individual countries was low, with the exception of the USA, which is considered as a region in its own right.

\section{Cost-effectiveness}

Trial-wide cost-effectiveness ratios are reported, as are regionspecific ratios, which were calculated by use of the regionspecific estimates of costs and number of QALYs described above. The method of recycled predictions [15] was employed to estimate costs and number of QALYs, and these were discounted at a rate of $3 \%$ per year. Cost and QALY number results are presented on the cost-effectiveness plane [16] for the US and Western European regions. The US region was chosen since this is the region with the highest healthcare costs, and the Western European region was chosen as being the region in which the majority of formal reimbursement authorities who make use of cost-effectiveness data reside. Incremental costeffectiveness ratios are presented in detail for the primary comparison of SFC versus $\mathrm{P}$ (the regulatory comparison) and for SFC versus Salm (since Salm is likely to be the standard of care in most countries).

\section{Uncertainty}

SEM were estimated by use of a nonparametric bootstrap, and 95\% confidence intervals (CIs) for incremental cost-effectiveness ratios were based on the bootstrap percentiles [17]. Costeffectiveness acceptability curves [18] were employed to represent the joint uncertainty across all four treatment options included in the TORCH trial for the US and Western European regions. In common with the majority of cost-effectiveness studies reported alongside clinical trials, the prices in the present analysis were treated as fixed weights. As a result, statistical measures of uncertainty in the cost of each treatment option relate to the sampling variability associated with the observed resource use in the study.

\section{RESULTS}

\section{Study population}

Table 1 shows the characteristics of the 4,237 participants in the TORCH study who were enrolled in the 21 countries for which validated translations of the EQ-5D instrument were available. The characteristics of this sample did not differ substantially from those of the full 6,112 patients reported in the TORCH study [3], except for the expected difference in racial mix associated with excluding the Asian/Pacific region and a lower proportion of males in the remaining four regions. The percentage of data points with missing resource use and EQ-5D information indicates that the problem of attrition was related to treatment group, with $\mathrm{P}$ showing the greatest quantity of missing data points and SFC the least. The number of deaths over the study period is also shown for the 4,237 participants. SFC resulted in the lowest number of deaths over the study period, although the reduction compared with $\mathrm{P}$ was nonsignificant at the $5 \%$ level.

\section{Cost and number of QALYs}

Table 2 shows trial-wide estimates of mean cost and number of QALYs for P, Salm, FP and SFC from the regression models, as well as estimates for each region. The mean cost of all three active treatment arms was greater than that of $\mathrm{P}$, with SFC 
TABLE 1 Characteristics of participants

\begin{tabular}{|c|c|c|c|c|c|}
\hline & All & $\mathbf{P}$ & Salm & FP & SFC \\
\hline Subjects $\mathrm{n}$ & 4237 & 1052 & 1055 & 1065 & 1065 \\
\hline Males \% & 70.7 & 71.3 & 71.3 & 69.6 & 70.5 \\
\hline Whites \% & 93.8 & 93.9 & 94.1 & 93.6 & 93.5 \\
\hline Body mass index $\mathrm{kg} \cdot \mathrm{m}^{-2}$ & $25.9 \pm 5.2$ & $26.0 \pm 5.2$ & $25.9 \pm 5.2$ & $25.9 \pm 5.2$ & $26.0 \pm 5.2$ \\
\hline Asia/Pacific & 0.0 & 0.0 & 0.0 & 0.0 & 0.0 \\
\hline Eastern Europe & 10.7 & 10.7 & 10.9 & 10.7 & 10.4 \\
\hline Western Europe & 38.2 & 38.3 & 38.2 & 38.3 & 38.1 \\
\hline Other & 18.3 & 18.2 & 18.1 & 18.3 & 18.7 \\
\hline Pre-BD FEV $1 \%$ pred & $40.8 \pm 11.9$ & $41.0 \pm 11.7$ & $39.9 \pm 12.1$ & $41.0 \pm 11.8$ & $41.3 \pm 12.0$ \\
\hline Current smokers \% & 43.6 & 44.1 & 43.6 & 43.4 & 43.5 \\
\hline \multicolumn{6}{|l|}{ Missing data \% } \\
\hline Resource use & 22.6 & 28.7 & 22.8 & 21.3 & 17.9 \\
\hline$E Q-5 D$ & 26.1 & 31.0 & 26.2 & 25.5 & 22.3 \\
\hline Died during study $\mathbf{n}(\%)$ & $555(12.8)$ & $145(13.8)$ & $133(12.6)$ & 155 (14.6) & $122(11.6)$ \\
\hline
\end{tabular}

Data are presented as mean \pm SD unless otherwise indicated. P: placebo; Salm: salmeterol; FP: fluticasone propionate; SFC: Salm/FP combination; pre-BD: prebronchodilator; FEV1: forced expiratory volume in $1 \mathrm{~s}$; SGRQ: St George's Respiratory Questionnaire; Exac: exacerbation; OCS: oral corticosteroid; EQ-5D: European quality of life five-dimension; \% pred: percentage of the predicted value.

being of significantly higher cost overall. Excluding study medications, COPD-related hospitalisations accounted for $47 \%$ of the remaining total cost, COPD medications $22 \%$, nonCOPD-related hospitalisations $22 \%$ and non-COPD-related medications the final 9\%. SFC-treated patients also gained significantly more QALYs than those receiving $\mathrm{P}(\mathrm{p}=0.002)$, Salm $(p=0.003)$ and FP $(p=0.03)$. There were no significant differences in numbers of QALYs between any of the other three therapy comparisons $(\mathrm{p}=0.30-0.75)$.

Table 2 also shows regional estimates. Joint tests of significance for treatment-by-region interactions in each of the four models revealed no evidence of heterogeneity across regions for treatment effects in the survival, EQ-5D or other cost (i.e. non-medication) regressions ( $\mathrm{p}>0.1$ for each test). However, evidence of heterogeneity in study medication cost was significant $(p<0.0001)$, with the costs in the USA being higher than those of other regions.

\section{Cost-effectiveness}

The trial-wide estimate of cost per QALY gained for the comparisons of SFC versus $\mathrm{P}$ and of SFC versus Salm is shown in table 3, together with cost-effectiveness estimates by region. SFC had a trial-wide point estimate of 43,600 USD compared

TABLE 2 Adjusted costs ${ }^{\#}$ and number of quality-adjusted life years (QALYs) for each arm of the TOwards a Revolution in COPD Health (TORCH) study by region and pooled across the whole trial

\begin{tabular}{|c|c|c|c|c|c|c|c|c|}
\hline & \multicolumn{2}{|c|}{$\mathbf{P}$} & \multicolumn{2}{|c|}{ Salm } & \multicolumn{2}{|c|}{ FP } & \multicolumn{2}{|c|}{ SFC } \\
\hline & Cost USD & QALYs n & Cost USD & QALYs n & Cost USD & QALYs n & Cost USD & QALYs n \\
\hline Eastern Europe & $4455 \pm 287$ & $1.863 \pm 0.040$ & $5815 \pm 294$ & $1.869 \pm 0.037$ & $6437 \pm 286$ & $1.904 \pm 0.037$ & $7710 \pm 300$ & $1.974 \pm 0.034$ \\
\hline Western Europe & $5623 \pm 324$ & $2.026 \pm 0.025$ & $6603 \pm 323$ & $2.030 \pm 0.024$ & $6797 \pm 318$ & $2.047 \pm 0.025$ & $7619 \pm 328$ & $2.109 \pm 0.023$ \\
\hline Other & $4490 \pm 354$ & $1.909 \pm 0.034$ & $5404 \pm 360$ & $1.919 \pm 0.032$ & $5726 \pm 350$ & $1.923 \pm 0.035$ & $6511 \pm 364$ & $2.003 \pm 0.032$ \\
\hline
\end{tabular}

Data are presented as mean \pm SEM. P: placebo; Salm: salmeterol; FP: fluticasone propionate; SFC: Salm/FP combination; USD: US dollars. ${ }^{\#}$ : translated into 2007 USD by means of purchasing power parity statistics. 
with P (95\% CI 21,400-123,500 USD). The trial-wide point estimates for Salm versus P (197,000 USD) and FP versus P (78,000 USD; data not shown) were less favourable, whereas the trial-wide cost-per-QALY estimates for SFC versus Salm (26,500 USD) and SFC versus FP (27,000 USD; data not shown) were necessarily more favourable. The cost-effectiveness estimates for all therapies in the USA were greater than those in other regions, reflecting the higher cost structure in the USA. In the USA, the point estimate for SFC versus $\mathrm{P}$ was 77,100 USD, which, again, was less than the US point estimates for Salm versus P (291,000 USD) and FP versus P (124,000 USD).

Ratios from the comparison of SFC versus Salm were lower than those from the comparison of SFC versus $\mathrm{P}$ because Salm produces fewer additional QALYs than does SFC, at a higher cost per QALY, and the same was true for the SFC versus FP comparison (results not shown, but available from the authors on request). This relationship is illustrated in figure 2 , which plots the incremental cost and number of QALYs gained for the three active treatments compared to $\mathrm{P}$ in the Western European region and in the USA. In these plots, the gradient of the line from the origin to the plotted point represents the point estimate for the cost-effectiveness ratio for the therapy compared to $\mathrm{P}$. The difference in the gradient of the line between the different active therapies represents the incremental cost-effectiveness ratio for the relevant therapy comparison. In both regions, Salm and FP produce fewer QALYs than does SFC, at a higher cost-per QALY ratio, compared to P, and this same pattern was apparent in the Eastern European and other regions (data not shown, but available from the authors on request).

Figure 3 shows the cost-effectiveness acceptability curves for the simultaneous comparison of uncertainty between the four arms of the TORCH trial in the Western European and US regions. These show the likelihood that each treatment arm is

\begin{tabular}{|c|c|c|c|}
\hline \multirow[t]{3}{*}{ TABLE 3} & \multicolumn{3}{|c|}{$\begin{array}{l}\text { Regional cost }{ }^{\#} \text { and number of quality-adjusted } \\
\text { life years (QALYs) for salmeterol (Salm)/ } \\
\text { fluticasone propionate combination (SFC) versus } \\
\text { placebo (P) and SFC versus Salm }\end{array}$} \\
\hline & \multicolumn{2}{|c|}{ Difference } & \multirow[t]{2}{*}{ Cost per QALY $(95 \% \mathrm{Cl})$ USD } \\
\hline & Cost USD & QALYs n & \\
\hline \multicolumn{4}{|l|}{ SFC versus $P$} \\
\hline USA & 5940 & 0.077 & 77100 (46200-241700) \\
\hline Eastern Europe & 3255 & 0.112 & $29100(18800-58700)$ \\
\hline Western Europe & 1996 & 0.083 & $24200(15200-56100)$ \\
\hline Other & 2021 & 0.094 & $21500(13400-60000)$ \\
\hline Pooled & 3511 & 0.081 & $43600(21400-123500)$ \\
\hline \multicolumn{4}{|l|}{ SFC versus Salm } \\
\hline USA & 3114 & 0.067 & $46300(28300-201300)$ \\
\hline Eastern Europe & 1895 & 0.105 & $18000(12200-37700)$ \\
\hline Western Europe & 1017 & 0.078 & $13000(8500-33100)$ \\
\hline Other & 1107 & 0.084 & $13200(8200-47000)$ \\
\hline Pooled & 1932 & 0.073 & $26500(7600-88800)$ \\
\hline
\end{tabular}

cost-effective for a given willingness to pay for an additional QALY. At a threshold willingness to pay for a QALY of 50,000 USD, the (one-tailed) likelihood for each of SFC, P, Salm and FP being cost-effective are $\sim 0.93, \sim 0.04, \sim 0.01$ and $\sim 0.03$, respectively, in the Western European region. Assuming a threshold willingness to pay in the USA of 100,000 USD per QALY gained, the corresponding likelihoods are $0.65,0.21,0.04$ and 0.10. The results for the Eastern European and other regions are similar to those of the Western European region (data not shown, but available from the authors on request).

\section{DISCUSSION}

The present article reports on the cost-effectiveness of ICSs and LABAs, alone or in combination, for the treatment of COPD, based on an economic analysis of the recently reported TORCH study [3]. The trial-wide point estimate for the costper-QALY ratio was 43,600 USD for combination therapy versus P (95\% CI 21,400-123,500 USD), but there was clear evidence of heterogeneity in study medication costs across regions, which limits the applicability of this overall result to the individual regions and countries that participated in the trial. When cost-effectiveness ratios were estimated for each region, it was found that, in the USA, the incremental cost-perQALY ratios for Salm, FP and SFC were all higher than those observed in other regions, reflecting the higher cost structure in the USA.

There is currently no single agreed methodology for the estimation of regional cost-effectiveness [19]. A recent suggestion [20] has been to use a formal heterogeneity test of costeffectiveness in order to choose between use of a single pooled estimate across the trial versus splitting the data and calculating region-specific cost-effectiveness ratios. Comparison of the pooled estimate reported here with four region-specific estimates based on splitting the data failed to reject the null hypothesis of homogeneity across regions (results not shown, but available from the authors on request). However, such an approach suffers from an acknowledged lack of power [20], which makes such negative test results difficult to interpret. The method reported here represents a variation on the approach suggested by COOK et al. [20], with heterogeneity tests conducted on individual components of the costeffectiveness calculation. Although the potential problem of lack of power remains, our preferred approach has the advantage that known differences in prices of medication between regions are not masked by high levels of uncertainty in other components of cost-effectiveness.

One of the challenges of interpretation of the economic evaluation of the TORCH trial is the simultaneous comparison of the four arms of the trial, as shown on the cost-effectiveness plane of figure 2. Incremental cost-per-QALY ratios involve the comparison of two alternatives. Since the incremental cost per QALY of SFC versus P is lower than that for Salm versus P or FP versus $\mathrm{P}$, the appropriate summary cost-effectiveness for SFC comes from the comparison with $\mathrm{P}$ (this is an example of the principle of extended dominance [21]). Although this is straightforward for point estimates, the analysis of uncertainty in cost-effectiveness results is less straightforward. The policy decision is to choose between all four options: withholding treatment, or use of either FP or Salm alone or in combination. One approach to expressing the uncertainty in this decision is 

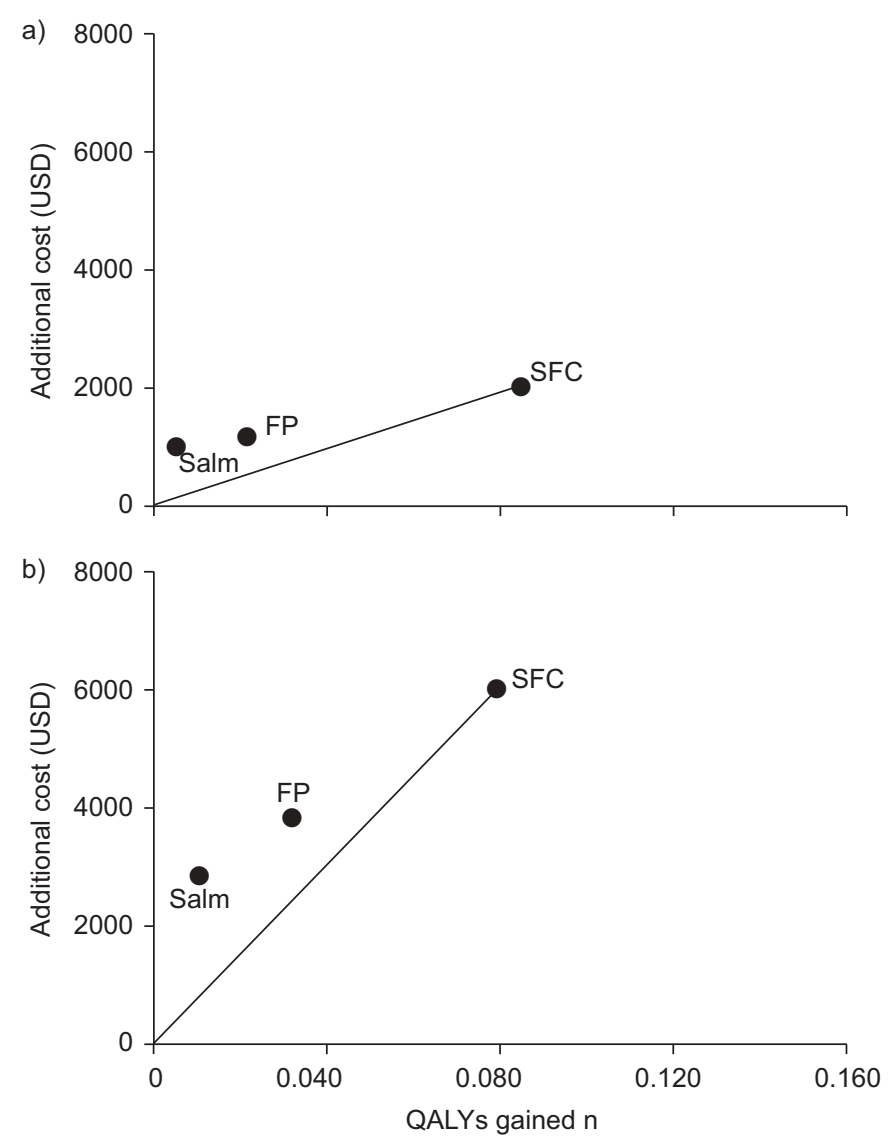

FIGURE 2. Cost-effectiveness plane for all three treatments (salmeterol (Salm), fluticasone propionate (FP) and Salm/FP combination (SFC)) compared to placebo for: a) the Western European region; and b) the US region. The gradient of the line connecting the SFC point estimate to the origin (placebo) represents the incremental cost-effectiveness of SFC. The Salm and FP point estimates lie above and to the left of this line, indicating that, although these options are of lower cost than SFC, the proportional increase in the number of quality-adjusted life years (QALYS) per additional US dollar (USD) spent was less than that of SFC. They are both, therefore, less efficient than SFC in producing QALYs given their cost.

represented by the multiple cost-effectiveness acceptability curves of figure 3 . Such presentations encourage the reader to think about the appropriate level of evidence that is sufficient to recommend adoption of a particular treatment rather than relying on arbitrary threshold p-values [22].

Another challenge faced in this analysis was the high levels of missing data for medical service costs $(22.6 \%)$ and preference scores $(26.1 \%)$ due to attrition during the study. The approach taken here was to use the inverse proportional weighting technique in combination with a set of multivariate regression models for the estimation of the components of the costeffectiveness calculation. This approach gives greater weight to the observed values of patients who have similar characteristics to those patients who have dropped out of the study. Since this approach is based on the observed data, it is unable to account for situations in which the propensity to be missing is directly related to the unobserved values (so-called nonignorable missingness). Nevertheless, if it is assumed that attrition in the study is associated with poor outcomes and
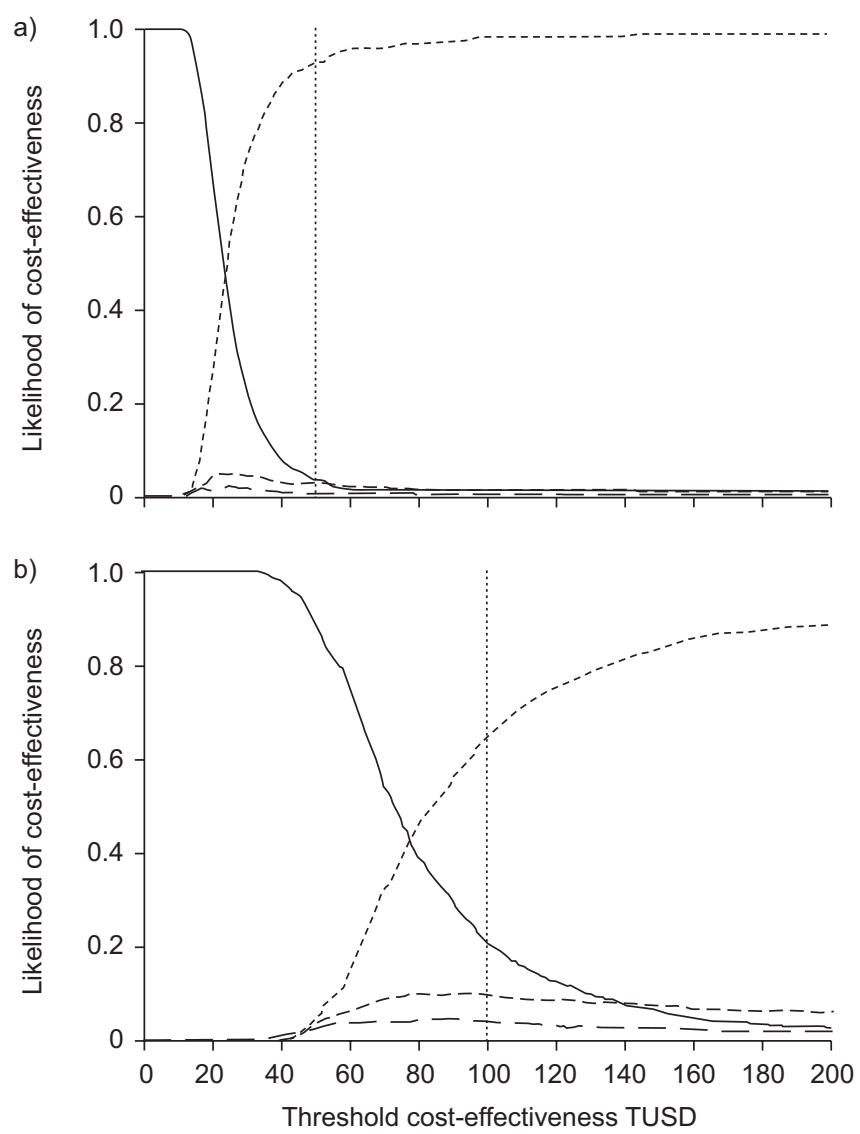

FIGURE 3. Cost-effectiveness acceptability curves for all treatment arms (_- placebo; — — —: salmeterol (Salm); - - - -: fluticasone propionate (FP); ------: Salm/FP combination) for: a) the Western European region; and b) the US region. Each curve represents the proportion of times in 1,000 bootstrap replications of the data that a treatment is preferred as a function of the threshold cost-effectiveness (ceiling cost-per-QALY ratio). The graph should be read vertically, with a given threshold cost-effectiveness being represented as a vertica line (..........; lines shown represent possible decision thresholds of 50 thousand US dollars (TUSD) in Western Europe and 100 TUSD in the USA). For each bootstrap replication at a particular threshold, one treatment is preferred. The proportion of times (across 1,000 replications) that each treatment is preferred is given by the cost-effectiveness likelihood at the intersection of the vertical line and the therapy curve. The sum of the four intersection likelihoods is 1.0

higher cost, and that the $\mathrm{P}$ group suffered the highest rates of attrition, the approach used in the present study is likely to be conservative with respect to estimating the value of treatment (versus P).

The scope of the resource use data collection in the TORCH study was limited by practical necessity. COPD-related costs were collected for medication and primary and secondary care resource categories. For non-COPD-related resource use, only secondary care (hospitalisations) was recorded. No rehabilitation data were collected, nor were any data collected on the costs borne by patients or carers, nor productivity losses to society. In practice, this limitation in scope is conservative with respect to the estimation of the cost-effectiveness of active treatment. Active treatments in the TORCH trial were shown to reduce COPD exacerbation rates compared to $\mathrm{P}$, with SFC 
achieving the greatest reduction. The analysis of all costs other than those related to study medication showed nonsignificant trends in favour of active treatment, and broadening the scope of cost data collection (particularly patient costs and productivity losses, which might be expected to be strongly correlated with exacerbations of COPD) would most probably have reinforced the study results in favour of active therapy.

The TORCH study has two great strengths. First, it is the first randomised controlled trial to be powered to examine mortality as a primary end-point. Secondly, it collected economic data prospectively within the trial, which permits the prospective assessment of value in terms of cost per QALY. Previous analyses of cost-effectiveness alongside clinical trials in COPD have only been able to report results in terms of cost per avoided exacerbation [23, 24], which does not facilitate comparisons of results across disease areas (required for reimbursement decisions). QALY number results have previously been presented for COPD treatments, but based on economic modelling studies, which typically project treatment effects from short-term randomised controlled trials over the lifetime of patients $[25,26]$. The cost-per-QALY results reported in the present article relate to the observed 3-yr duration of follow-up of the TORCH study. Relatively simple models projecting this experience beyond the 3-yr results suggest that cost-effectiveness ratios for long-term therapy are likely to fall by $\sim 50 \%$ after 6 yrs of projection (i.e. after the ninth year of therapy), in line with other studies in which benefits have been reported first over the period of the trial and then extrapolated to patient lifetimes [27-30]. Therefore, the within-trial results presented here are likely to represent a conservative estimate of the true cost-effectiveness of longterm COPD treatment.

Ultimately, it will be for individual countries to judge whether the combined ICS and LABA therapy represents a costeffective use of resources within their own jurisdiction, given the point estimates of cost-effectiveness and associated uncertainty. In the UK, for example, it is commonly regarded that treatments costing $<20,000$ pounds sterling (GBP) per QALY are likely to be considered cost-effective, whereas those of $>30,000 \mathrm{GBP}$ are unlikely to be acceptable on cost-effectiveness grounds alone [31]. In the USA, there is no single reimbursement authority; nevertheless, a threshold of 50,000 USD per QALY is commonly quoted, and, more recently, commentators have suggested that a contemporary threshold is likely to be $\geqslant 100,000$ USD [32]. In comparison to other disease areas, the cost-effectiveness of SFC therapy falls somewhere between primary prevention of cardiovascular disease with statins [33] and secondary prevention with implantable defibrillators [34], both of which have received positive recommendations from the National Institute for Health and Clinical Excellence in the UK.

To summarise, based on the analysis of data from the TORCH study, SFC is more effective and has a lower incremental costeffectiveness ratio (compared with P) than either FP or Salm used alone, and, therefore, this combination therapy is preferred to the existing use of monotherapy on efficiency grounds. For patients who are treatment-naive, whether combination therapy offers good value for money is a judgment for the decision-makers in each jurisdiction. Nevertheless, the present economic analysis of the TORCH trial provides a strong empirical basis for making those decisions.

\section{SUPPORT STATEMENT}

Financial support was provided by GlaxoSmithKline.

\section{STATEMENT OF INTEREST}

Statements of interest for all of the authors and the study itself can be found at www.erj.ersjournals.com $/ \mathrm{misc} /$ statements.dtl

\section{REFERENCES}

1 Soler JJ, Martinez-Garcia MA, Roman P, et al. Severe acute exacerbations and mortality in patients with chronic obstructive pulmonary disease. Thorax 2005; 60: 925-931.

2 Petty TL. Definition, epidemiology, course, and prognosis of COPD. Clin Cornerstone 2003; 5: 1-10.

3 Calverley PMA, Anderson JA, Celli B, et al. Salmeterol and fluticasone propionate and survival in chronic obstructive pulmonary disease. $N$ Engl J Med 2007; 356: 775-789.

4 Quanjer PH, Tammeling GJ, Cotes JE, et al. Lung volumes and forced ventilatory flows. Eur Respir J 1993; 6: Suppl. 16, 5-40.

5 The EuroQol Group. EuroQol - a new facility for the measurement of health-related quality of life. Health Policy 1990; 16: 199-208.

6 Dolan P. Modeling valuations for EuroQol health states. Med Care 1997; 35: 1095-1108.

7 Wickersham RM, Novak KK, eds, Drug Facts and Comparisons. St Louis, Wolters Kluwer Health, Inc., 2007.

8 Organization for Economic Co-operation and Development, Purchasing power parities. Main Economic Indicators 2007; 2007: 278-279.

9 World Health Organization. Purchasing Power Parity 2000. www. who.int/choice/costs/ppp/en/ Date last accessed: March 6, 2008.

10 The Economist. The Big Mac index. The Economist. February 1, 2007. www.economist.com/markets/indicators/displaystory. cfm?story_id $=8649005 \& C F I D=13546391 \& C F T O K E N=b 6489205 b$ a0e3306-85B21FBD-B27C-BB00-01296499DCFD7106 Date last accessed: March 6, 2008

11 Fletcher CM. Standardised questionnaire on respiratory symptoms: a statement prepared and approved by the MRC Committee on the Aetiology of Chronic Bronchitis (MRC breathlessness score). BMJ 1960; 2: 1665.

12 Jones PW, Quirk FH, Baveystock CM, et al. A self-complete measure of health status for chronic airflow limitation: the St George's Respiratory Questionnaire. Am Rev Respir Dis 1992; 145: 1321-1327.

13 Lin DY. Linear regression analysis of censored medical costs. Biostatistics 2000; 1: 35-47.

14 Lin DY. Regression analysis of incomplete medical cost data. Stat Med 2003; 22: 1181-1200.

15 Glick HA, Doshi JA, Sonnad SS, et al. Economic Evaluation in Clinical Trials. Oxford, Oxford University Press, 2007.

16 Black WC. The cost-effectiveness plane: a graphical representation of cost-effectiveness. Med Decis Making 1990; 10: 212-214.

17 Briggs AH, Wonderling DE, Mooney CZ. Pulling cost-effectiveness analysis up by its bootstraps: a non-parametric approach to confidence interval estimation. Health Econ 1997; 6: 327-340.

18 Fenwick E, Claxton K, Sculpher M. Representing uncertainty: the role of cost-effectiveness acceptability curves. Health Econ 2001; 10: 779-787.

19 Reed SD, Anstrom KJ, Bakhai A, et al. Conducting economic evaluations alongside multinational clinical trials: toward a research consensus. Am Heart J 2005; 149: 434-443.

20 Cook JR, Drummond MR, Glick H, et al. Assessing the appropriateness of combining economic data from multinational clinical trials. Stat Med 2003; 22: 1955-1976. 
21 Cantor S. Cost-effectiveness analysis, extended dominance, and ethics: a quantitative assessment. Med Decis Making 1994; 14: 259-265.

22 Claxton K, Sculpher MJ, Drummond M. A rational framework for decision making by the National Institute for Clinical Excellence (NICE). Lancet 2002; 360: 711-715.

23 Oostenbrink JB, Rutten-van Mölken MP, Al MJ, et al. One-year cost-effectiveness of tiotropium versus ipratropium to treat chronic obstructive pulmonary disease. Eur Respir J 2004; 23: 241-249.

24 Rutten-van Mölken MP, van Nooten FE, Lindemann M, et al. A 1year prospective cost-effectiveness analysis of roflumilast for the treatment of patients with severe chronic obstructive pulmonary disease. Pharmacoeconomics 2007; 25: 695-711.

25 Oostenbrink JB, Rutten-van Mölken MP, Monz BU, et al. Probabilistic Markov model to assess the cost-effectiveness of bronchodilator therapy in COPD patients in different countries. Value Health 2005; 8: 32-46.

26 Spencer M, Briggs AH, Grossman RF, et al. Development of an economic model to assess the cost effectiveness of treatment interventions for chronic obstructive pulmonary disease. Pharmacoeconomics 2005; 23: 619-637.

27 Kim LG, Thompson SG, Briggs AH, et al. How cost-effective is screening for abdominal aortic aneurysms? J Med Screen 2007; 14 46-52.

28 Mihaylova B, Briggs A, Armitage J, et al. Cost-effectiveness of simvastatin in people at different levels of vascular disease risk: a randomised trial in 20,536 high-risk individuals. Lancet 2005; 365 1779-1785.

29 Mihaylova BN. Using clinical trial evidence for economic evaluation of health care interventions. DPhil thesis. University of Oxford, Oxford, UK, 2006.

30 Glick H, Cook J, Kinosian B, et al. Costs and effects of enalapril therapy in patients with symptomatic heart failure: an economic analysis of the Studies of Left Ventricular Dysfunction (SOLVD) Treatment Trial. J Card Fail 1995; 1: 371-380.

31 Rawlins MD, Culyer AJ. National Institute for Clinical Excellence and its value judgments. BMJ 2004; 329: 224-227.

32 Braithwaite SR, Meltzer D, King J, et al. What does the value of modern medicine say about the $\$ 50,000$ per quality-adjusted lifeyear decision rule? Med Care 2008; 46: 349-356.

33 National Institute for Health and Clinical Excellence. Statins for the Prevention of Cardiovascular Events. Technology Appraisal 94. London, National Institute for Health and Clinical Excellence, 2006. www.nice.org.uk/TA094 Date last updated: January 2006. Date last accessed: October 17, 2008.

34 National Institute for Health and Clinical Excellence. Implantable Cardioverter Defibrillators for Arrhythmias: Review of Technology Appraisal 11. Technology Appraisal 95. London, National Institute for Health and Clinical Excellence, 2007. www.nice.org.uk/TA095 Date last updated: July 2007. Date last accessed: October 17, 2008. 